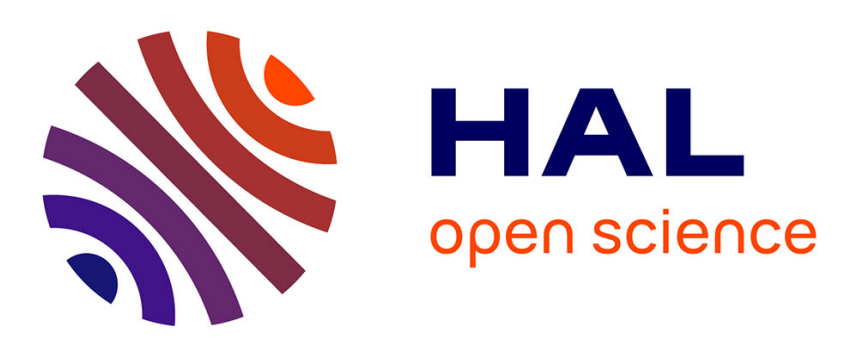

\title{
Molecular dynamics of liquid benzene via femtosecond pulses laser excitation
}

J. Etchepare, G. Grillon, G. Hamoniaux, A. Antonetti, A. Orszag

\section{To cite this version:}

J. Etchepare, G. Grillon, G. Hamoniaux, A. Antonetti, A. Orszag. Molecular dynamics of liquid benzene via femtosecond pulses laser excitation. Revue de Physique Appliquée, 1987, 22 (12), pp.17491753. 10.1051/rphysap:0198700220120174900 . jpa-00245735

\section{HAL Id: jpa-00245735 https://hal.science/jpa-00245735}

Submitted on 1 Jan 1987

HAL is a multi-disciplinary open access archive for the deposit and dissemination of scientific research documents, whether they are published or not. The documents may come from teaching and research institutions in France or abroad, or from public or private research centers.
L'archive ouverte pluridisciplinaire HAL, est destinée au dépôt et à la diffusion de documents scientifiques de niveau recherche, publiés ou non, émanant des établissements d'enseignement et de recherche français ou étrangers, des laboratoires publics ou privés. 


\title{
Molecular dynamics of liquid benzene via femtosecond pulses laser excitation
}

\author{
J. Etchepare, G. Grillon, G. Hamoniaux, A. Antonetti and A. Orszag \\ Laboratoire d'Optique Appliquée, E.N.S. Techniques Avancées, Ecole Polytechnique, Batterie de l'Yvette, \\ 91120 Palaiseau, France
}

(Reçu le 12 juin 1987, accepté le 18 septembre 1987)

\begin{abstract}
Résumé. - L'étude temporelle de l'évolution des réseaux induits associée à une analyse de la polarisation du faisceau diffracté, a permis de séparer dans $\mathrm{C}_{6} \mathrm{H}_{6}$ liquide plusieurs composantes de polarisation induite : un phénomène de type électronique et trois phénomènes ayant leur origine dans la variation de la polarisabilité associée aux mouvements moléculaires. L'étude des réponses temporelles conduit à considérer un temps d'établissement de l'anisotropie induite par les mouvements moléculaires.
\end{abstract}

\begin{abstract}
We analyse the complex response of liquid benzene to the applied 45 fs FHWM optical pulses by using a polarization sensitive transient grating technique. An electronic contribution to the nonlinear polarization is distinguished from three other processes which, we demonstrate, originate in polarizability modifications associated to molecular motions. Additionally, we present evidence that orientation of $\mathrm{C}_{6} \mathrm{H}_{6}$ molecules towards the applied optical field is not instantaneous. The time it takes for the molecules to orient along the incident field is measured.
\end{abstract}

\section{Introduction.}

Molecular reorientation in liquids has been studied extensively in the past by depolarized Rayleigh scattering. These numerous studies, combined with nuclear magnetic resonance spin-lattice relaxation results, lead to a determination of reorientation times along different molecular axes. In recent years, spontaneous scattering techniques have been superseded by nonlinear optical techniques due to the dramatic development of ultra-short and/or highly tunable optical pulses; thus leading to a direct determination of temporal characteristics or of their Fourier transform analog. As an example, optical Kerr effect technique, one of the most efficient methods for studying molecular reorientation directly in the time domain, has a temporal resolution of tens of femtoseconds. This resolution enhancement which results from utilization of tens of fs FWHM pulses, may give access to orientation rise time of molecules. In this respect, C. Kalpouzos et al. argued for a «delay » in the molecular responses, to explain the femtosecond Kerr signal in liquid $\mathrm{CS}_{2}$ [1]. In a parallel direction, we have already used a polarization sensitive transient grating technique to extensively determine the nonlinear susceptibility tensors which describe the dynamics of liquid $\mathrm{CS}_{2}$ [2], leading therefore to a measure of the time it takes for the molecules to move towards the driving optical field. We report here new results obtained by the transient grating temporal behaviour analysis of benzene, a molecule for which rapid molecular motions are known to relax on a time scale even shorter than in the case of $\mathrm{CS}_{2}$.

\section{Description of the technique.}

We briefly report here a sketch of our transient grating experiment [2], which by a specific polarization analysis allows an optimal temporal discrimination between processes of different physical origin. In liquids, the third order nonlinear susceptibility arises from two different phenomena : the electronic hyperpolarizability which has its origin in the deformation of electronic clouds surrounding the atoms of each molecule, and the orientational motions of molecules which are connected to a variation in their polarizability. Both kinds of processes lead, therefore, to a transient anisotropy. In the specific case of transparent media, the relationships between the associated tensor elements are quite different; we then can use specific polarization states for the 
incoming pulses in order to discriminate between them, and particularly vary their mutual contribution to the overall signal.

Consider two input beams of frequency $w_{1}$ and polarization along $\hat{x}$, propagating in an isotropic medium. They create a transient grating which will diffract a third beam of frequency $w_{2}$ and polarization along a direction at an angle $\alpha$ with $\hat{x}$. If we detect the diffracted signal at frequency $w_{2}$, by using an analyser along $\hat{X}$, which makes an angle $\beta$ with respect to $\hat{x}$, the output intensity will be given by :

$$
\begin{aligned}
I_{X} \propto\left[\sum _ { i } \left(X_{x x x x, i} \cos \alpha \cos \beta+\right.\right. \\
\left.\left.+X_{y y x x, i} \sin \alpha \sin \beta\right) E_{2} E_{1} E_{1}^{*}\right]^{2} \\
I_{X} \propto\left[\left(\sum_{i} X_{\mathrm{eff} . i}\right) E_{2} E_{1} E_{1}^{*}\right]^{2}
\end{aligned}
$$

where the summation is performed over the $i$ processes involved. Molecular and electronic processes being characterized by different relationships between $X_{x x x x}$ and $X_{y y x x}$ coefficients, their specific contribution will be cancelled for different values of $\beta$ : in the case where $\alpha=45^{\circ}$, these $\beta$ values are respectively $63^{\circ} 26^{\prime}$ and $-71^{\circ} 56^{\prime}$.

Extension of equations 1 to a temporal analysis is straightforward. As a general result, one can very precisely study the kinetics of short-lived processes, by suppressing the electronic contribution, and temporally resolve the primary events with a high resolution. On the contrary, cancellation of all molecular contributions leads to a direct measurement of the electronic nonlinearity. Furthermore, in the case of nonresonant processes, knowledge about the instrumental response function can be obtained through the third order correlation between pump squared and probe pulses, and the zero time delay can be defined unambiguously.

\section{Experimental arrangement.}

The experiments were performed using a CPM dye laser that produces pulses centred at $620 \mathrm{~nm}$ and amplified at $10 \mathrm{~Hz}$ repetition using a four stage amplifier. Two sets of four prisms, one inside the oscillator and the other after the amplifier, ensure that we obtain Fourier transform limited $45 \mathrm{fs}$ FWHM pulses [3]. Pump pulses, at frequency $w_{1}=620 \mathrm{~nm}$, are impinging, at an angle of 1-3 degrees with respect to each other, on a $1 \mathrm{~mm}$ thick sample. Probe pulses are generated at frequency $w_{2}=650 \mathrm{~nm}$ by interaction in a $1 \mathrm{~mm}$ water cell and insertion of an interference filter of adequate spectral width; particular care was taken in order not to lengthen the pulse during spectral conversion. Use of a different wavelength for the probe pulse leads to two essential advantages. First, only the grating created by $w_{1}$ pulses is present, due to the fact that $w_{1}$ and $w_{2}$ are not mutually coherent. Second, it allows a better discrimination of the scattering of the pump pulses into the probe direction; therefore it leads to a higher dynamics for the signal. A nonplanar, Bragg configuration was chosen for the diffracted geometry ; it permits a maximum efficiency of the grating, which allows the use of moderate peak intensities for the pump pulses $\left(5 \mathrm{GW} / \mathrm{cm}^{2}\right)$. This is a necessary condition not to perturb significantly the signal polarization state by the inherent phase shift encountered by two orthogonal components of the probe pulse polarization, along and perpendicular to the pump pulse polarization direction [4]. Input beam polarization directions are obtained by propagation through Glan-air prisms. We chose for the probe beam polarization direction, $\alpha=45^{\circ}$. The specific values of $\beta$ give access to all the coefficients of $X^{(3)}$ tensors. Other polarization configurations are possible, in particular crossed polarization for the two pump pulses ; in this case, one can suppress contributions coming from amplitude grating, but has only access to $\left(X_{x y x y}+X_{x y y x}\right)$ combination. The diffracted beam is transmitted through a Wollaston prism mounted on a rotating plate : it allows simultaneous analysis, as a function of angle $\beta$, of two perpendicularly polarized components of the signal. One main advantage of our apparatus is the fixed position of the zero time delay between pump and probe pulses, for various polarizations of diffracted signal. This is due to the fact that in order to measure different $X_{i j k l}$ coefficients, only the Wollaston prism is rotated, leaving the position of $t=0$ unchanged. Furthermore, possible variations in the energy density of the pulses for experiments at different values of $\beta$ may be accounted for by a normalization through the overall diffracted intensity which remains constant.

\section{Discussion of the results.}

Examination of previous studies on orientational relaxation and induced nonlinear polarizations of $\mathrm{C}_{6} \mathrm{H}_{6}$ molecule $[5,6]$, provides an interesting background for interpreting our results. First of all, benzene molecules are characterized by a high electronic nonlinearity which has its origin in the delocalization of electrons along the aromatic ring. The Kleinman symmetry rule [7] seems to be obeyed, in the limit of the accuracy of available data. The symmetry associated to the molecule $\left(D_{6 h}\right)$ allows two orientational motions. The one perpendicular to the highest symmetry axis $\left(\mathrm{C}_{6}\right)$, generates a variation of the polarizability with the longest relaxation time of around $3 \mathrm{ps}$. The other (degenerate) rotational motion does not lead to any modification of the polarizability and its activity should therefore be forbidden : extrapolation from 
NMR measurements gives a temporal value of 0.7 ps. Recent works in the temporal domain $[8,9]$ reveal also the existence of a more rapid motion, with a characteristic time $<0.2 \mathrm{ps}$, with its origin related to the librational motions activated by interaction induced processes [10]. Other interesting experimental data show polarizability of $\mathrm{C}_{6} \mathrm{H}_{6}$ molecules to be smaller than that of $\mathrm{CS}_{2}$ [11], resulting in a smaller contribution to the induced polarization.

We have reproduced in figures 1 to 4 several characteristic results concerning specific orientations of the Wollaston prism. As a general feature, polarization selection rules are fully consistent with the existence of only two physically different processes : an electronic one in which maximum and null contributions correspond to theoretically expected $\beta$ values; and three molecular processes, which obey the same law based on an ellipsoid form of the polarizability.

Study of temporal decrease of the induced grating lead to the determination of the three characteristic relaxation times : $3.0 \pm 0.1 \mathrm{ps}, 0.6 \pm 0.1 \mathrm{ps}$ and $0.06 \pm 0.01 \mathrm{ps}$ respectively. The first one is undoubtedly attributed to orientational relaxation time of individual molecules, with a rotational motion perpendicular to the $\mathrm{C}_{6}$ axis. The second one has the

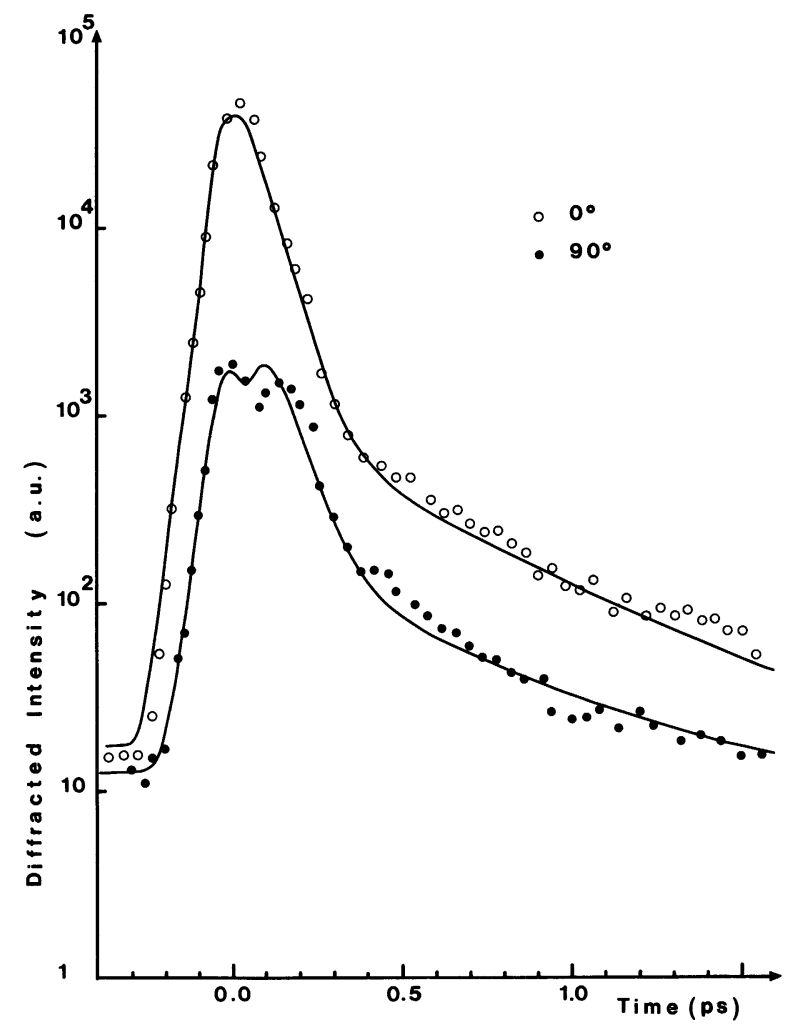

Fig. 1. - Diffracted intensity, as a function of temporal delay between pumps and probe pulses, of the induced grating created in a $1 \mathrm{~mm}$ cell of $\mathrm{C}_{6} \mathrm{H}_{6} . \mathrm{O}$ and $\bullet$ correspond to $X_{1111}$ and $X_{2211}$ coefficients.

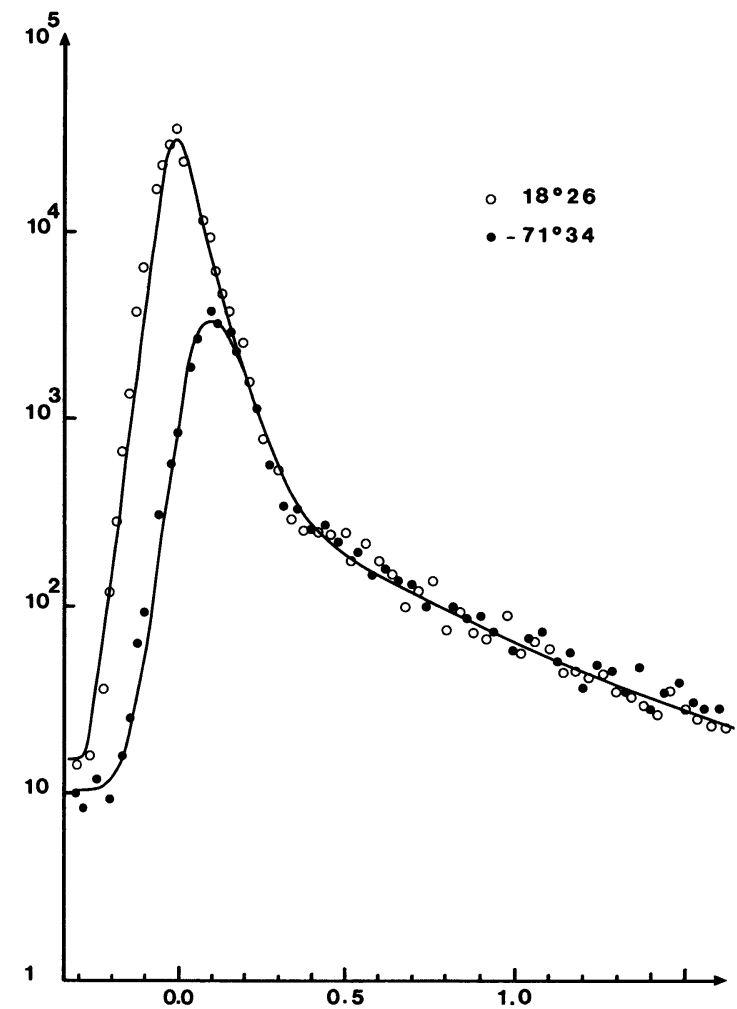

Fig. 2. - Diffracted intensity, as a function of temporal delay between pumps and probe pulses, of the induced grating created in a $1 \mathrm{~mm}$ cell of $\mathrm{C}_{6} \mathrm{H}_{6}$. $\mathrm{O}$ and $\bullet$ correspond to maximum and null contribution of electronic process.

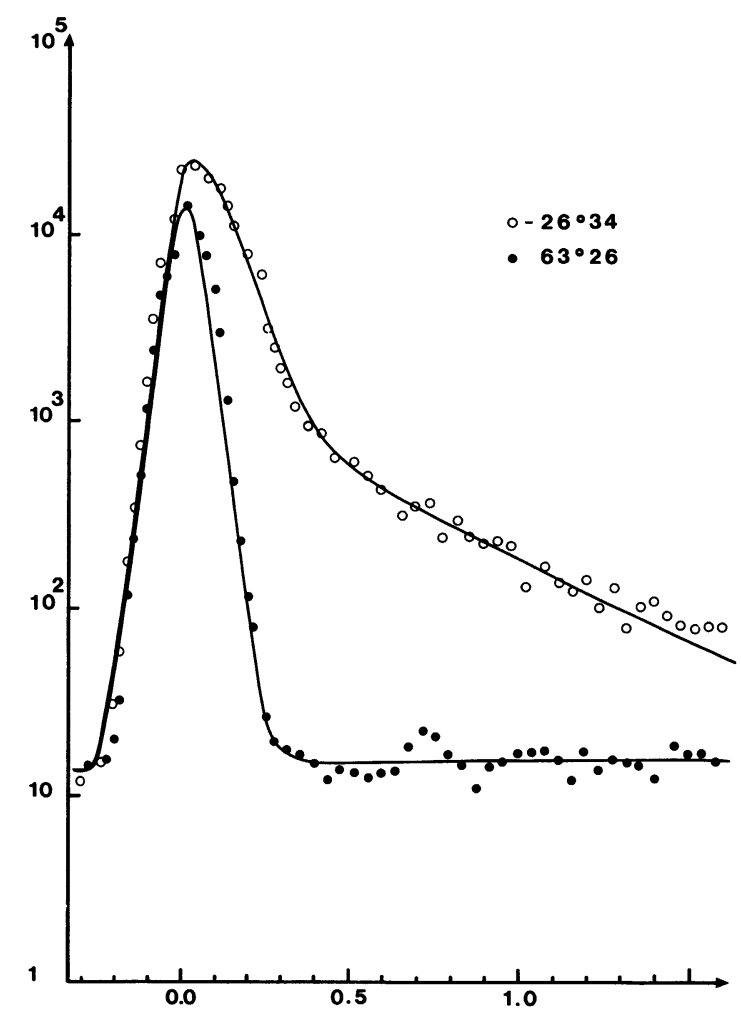

Fig. 3. - Diffracted intensity, as a function of temporal delay between pumps and probe pulses, of the induced grating created in a $1 \mathrm{~mm}$ cell of $\mathrm{C}_{6} \mathrm{H}_{6} . \mathrm{O}$ and $\bullet$ correspond to maximum and null contributions of molecular processes. 


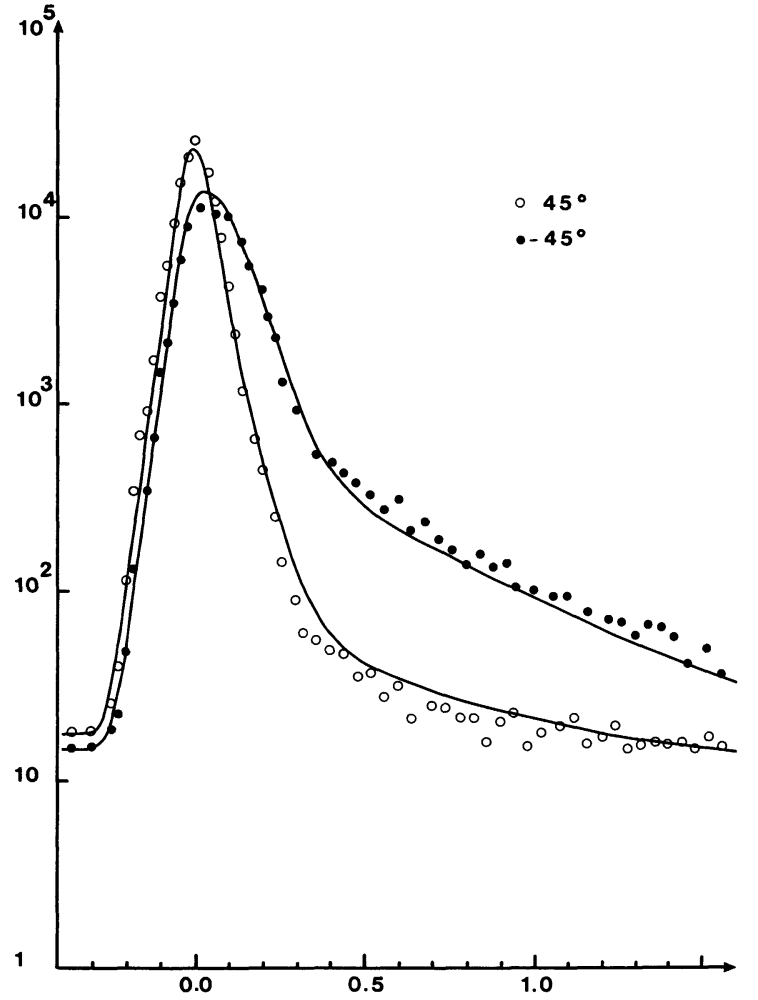

Fig. 4. - Diffracted intensity, as a function of temporal delay between pumps and probe pulses, of the induced grating created in a $1 \mathrm{~mm}$ cell of $\mathrm{C}_{6} \mathrm{H}_{6}$. $\bullet$ configuration corresponds to Kerr experimental results : $X_{1212}+X_{1221}$.

same value as the degenerate rotational motion ; if it corresponds effectively to such a motion, this interpretation would support a breakdown of symmetry rules due to interaction between molecules which are not distributed spherically, but distributed with a packing similar to crystalline arrangement. Existence of similar behaviour for nonsymmetrically substituted benzene molecules withstands this hypothesis. The interaction induced process is characterized by the third relaxation time, a value even shorter than that of $\mathrm{CS}_{2}$ molecules.

The most interesting result concerns the appearance of a delay which is quite visible for the fastest motion. This delay could be observed clearly because of three effects : (1) very sharp temporal slopes of pump and probe pulses which result in a high temporal resolution, (2) comparison with an instantaneous process which allows a high accuracy determination of zero time delay, and (3) existence of different susceptibility tensor characteristics, leading, especially at $90^{\circ} \beta$ value, to an interference phenomenon which increases the splitting between electronic and interaction induced processes. Modeling of this behaviour has been attempted in several ways. To describe their optical Kerr effect results on $\mathrm{CS}_{2}$ [1] and benzene derivatives [12], these authors plainly shift temporally the exponentially decaying functions which describe molecular motions and sum these forced delayed signals to the electronic instantaneous response. On the contrary, we chose to model this delay by a noninstantaneous motion of molecules towards the direction of the applied field, say by an exponential function : $\exp \left(-t / \tau_{\mathrm{R}}\right)$. Consequently, the population of an orientationally excited molecule takes the form: $\exp \left(-t / \tau_{\mathrm{D}}\right)-$ $\exp \left(t / \tau_{\mathrm{R}}\right)$, with relaxation time $\tau_{\mathrm{D}}$. In a preliminary approach, we have also assumed that the various orientational motions were independent. Convolution of the $i$ th orientationally excited state's temporal evolution with the applied pump pulses describes finally the kinetics of the induced gratings :

$$
\begin{aligned}
S(t)=\int_{-\infty}^{t} \sum_{i} X_{\mathrm{eff}, i} \tau_{\mathrm{D}, i} /\left(\tau_{\mathrm{D}, i}-\tau_{\mathrm{R}, i}\right) \\
\times I_{\mathrm{p}}\left(t^{\prime}\right)\left[\exp -\left(t-t^{\prime}\right) / \tau_{\mathrm{D}, i}\right. \\
\left.-\exp -\left(t-t^{\prime}\right) / \tau_{\mathrm{R}, i}\right] \mathrm{d} t^{\prime}
\end{aligned}
$$

The diffracted intensity is therefore given by :

$$
I(t) \propto \int_{-\infty}^{+\infty} I_{t}\left(t^{\prime}\right) S\left(t-t^{\prime}\right) \mathrm{d} t^{\prime}
$$

Fitting of experimental curves results in the determination of $X_{1111, i}$ nonlinear parameters and $\tau_{\mathrm{D}, i}$ and $\tau_{\mathrm{R}, i}$ temporal characteristics. Results are gathered in table I. It appears that the best fits correspond to roughly equal values of $\tau_{D}$ and $\tau_{R}$ in the case of the fastest process. The other processes have also been fitted with this same value for $\tau_{R}$, but its contribution does not really increase the quality of the adjustments. Concerning this temporal behaviour description, more elaborate equations have been proposed in the past $[13,14]$. Whatever the mechanism for the fastest process, inertial effects are taken into account, resulting in a second order differential equation for the temporal behaviour description of the nonlinear refractive index as a function of field amplitude. This still leads to the determination of two characteristic times, which also have similar values [13]. On the other hand, values obtained for $X_{1111, i}$ coefficients are convincing, especially with regard to the electronic contribution which is, as expected, higher than in the case of $\mathrm{CS}_{2}$, but still remains in a usually admitted range [6].

Table I.

\begin{tabular}{llll}
\hline Process & \multicolumn{2}{c}{$\begin{array}{c}\text { Characteristic times (ps) } \\
\text { rise time }\end{array}$} & $\begin{array}{c}X_{1111} \\
\text { relaxation time }\end{array}$ \\
\hline \multicolumn{2}{c}{ nonmeasurables }
\end{tabular}

Third order nonlinearity parameters for $\mathrm{C}_{6} \mathrm{H}_{6}$ molecule 


\section{Conclusion.}

In this paper, we have shown that the polarization analysis of a transient grating experiment may give important information on molecular dynamics in fluids. Novel interesting results have been obtained on liquid benzene, where three noninstantaneous processes related to molecular motions have been clearly observed. It is shown that it takes some time for the molecules to orient themselves towards the driving field. This orientation of molecules stays on even when the pump pulses are no longer present, a behaviour which we are currently investigating by examination of inertial and frictional effects in related molecules. We also resolved the only instantaneous contribution, which comes from the electronic part of the third order susceptibility tensor ; we thereby have taken advantage of a precise determination of zero time delay and of the apparatus function to reach the femtosecond resolution in our measurements.

\section{References}

[1] Kalpouzos, C., Lotshaw, W. T., McMorrow, D. and Kenney-Wallace, G. A., J. Chem. Phys. 91 (1987) 2028.

[2] Etchepare, J., Grillon, G., Chambaret, J. P., Hamoniaux, G. and Orszag, A., Opt. Commun. 63 (1987) 329.

[3] Valdmanis, J. A., Fork, R. L. and Gordon, J. P., Opt. Lett. 10 (1985) 131 ; FORK, R. L., MARTINEZ, O. E. and Gordon, J. P., Opt. Lett. 9 (1984) 150.

[4] Etchepare, J., Grillon, G., Chambaret, J. P. and ANTONETTI, A., submitted.

[5] Bauer, D. R., Alms, G. R., Brauman, J. I. and Pecora, R., J. Chem. Phys. 61 (1974) 2255 and references therein.

[6] Levenson, M. D. and Bloembergen, N., Phys. Rev. B 10 (1974) 4447.

[7] Kleinman, D. A., Phys. Rev. 126 (1962) 1977.
[8] Tang, C. L. and Halbout, J. M., in Picosecond Phenomena III (Springer-Verlag) 1982, p. 212.

[9] Etchepare, J., Grillon, G., Astier, R., Martin, J. L., Bruneau, C. and Antonetti, A., in Picosecond Phenomena III (Springer-Verlag) 1982, p. 217.

[10] Madden, P. A., in Ultrafast Phenomena IV (Springer-Verlag) 1984, p. 244.

[11] Alms, G. R., Burnham, A. K. and Flygare, W. H., J. Chem. Phys. 63 (1975) 3321.

[12] Lotshaw, W. T., McMorrow, D., Kalpouzos, C. and Kenney-Wallace, G., Chem. Phys. Lett. 136 (1987) 323.

[13] Cubeddu, R., Polloni, R., Sacchi, C. A. and Svelto, O., Phys. Rev. A 2 (1970) 1955.

[14] McConnel, J., in Rotational Brownian Motion and Dielectric Theory (Academic Press) 1980. 